\title{
VINGT-CINQ ANS DE RECHERCHES SUR LA VERSION ARABE DE GRÉGOIRE DE NAZIANZE A LOUVAIN-LA-NEUVE
}

On sait que c'est à l'occasion des manifestations jubilaires organisées à Coblence en 1976, par la Görres-Gesellschaft, qu'un Symposium Nazianzenum avait permis de commencer le programme d'editio major des œuvres de Grégoire le Théologien. Après l'approbation de ce programme par l'assemblée générale de la Görres-Gesellschaft à Nuremberg en 1971, il fut décidé que le Professeur M. Sicherl, de l'Université d'Etat de Münster, accompagné d'une équipe de cette université, se consacrerait à l'édition des Carmina, tandis qu'une équipe de l'Institut Orientaliste de l'Université catholique de Louvain à Louvain-la-Neuve, se chargerait de l'édition critique des Discours.

En 1980 il fut décidé que cette équipe d'orientalistes et d'hellénistes allait constituer ,un groupe de travail pour l'étude des Discours de Grégoire de Nazianze dans leur texte original et dans leurs versions orientales anciennes conformément au programme patronné par la Görres-Gesellschaft"1. En 1981, un deuxième Symposium Nazianzenum ${ }^{2}$ se réunit à Louvain-la-Neuve autour des promoteurs du projet et les Actes, publiés en 1983, contiennent les ,premières tranches de traductions orientales éditées pour la première fois (arabe: Homélie 24, Sur Cyprien ${ }^{3}$; arménien: une homélie apocryphe), le répertoire complet des manuscrits géorgiens de Grégoire le Théologien, et plusieurs études préliminaires sur la version syriaque, sur les textes grecs et sur les commentaires"4.

${ }^{1}$ Cfr. B. Coulie (éd.), Versiones orientales, repertorium ibericum et studia ad editiones curandas, CCG 20, Corpus Nazianzenum 1, Turnhout 1988, p. VIII (= Coulie, Versiones Orientales).

${ }^{2}$ Cfr. II. Symposium Nazianzenum (Louvain-la-Neuve, 25-28 août 1981). Actes édités par Justin Mossay (Studien zur Geschichte und Kultur des Altertums, Neue Folge, 2. Reihe: Forschungen zu Gregor von Nazianz), Paderborn - München - Wien - Zürich 1983.

${ }^{3}$ Cfr. J. Grand'Henry (éd.), La version arabe du discours 24 de Grégoire de Nazianze, édition critique, commentaires et traduction, dans: Coulie, Versiones orientales, p. 197-291 (= Grand'Henry, Discours 24 arabe).

${ }^{4}$ Cfr. G. Garitte, Avant-propos, dans: Coulie, Versiones orientales, p. VI. 
Parmi les manuscrits qui constituent la base de cette édition, certains ont des colophons qui apportent des éléments intéressants pour reconstruire l'histoire du texte arabe: ainsi par exemple, le manuscrit $A$ (= Patriarcat copte du Caire 617, theol. 116, a. 1231) a un colophon écrit par le moine copiste Zacharie, nom qui se retrouve aussi dans le manuscrit Paris syr. 191 et fait référence au monastère de saint Antoine dans le désert oriental égyptien de la Araba. Zacharie y signale que le recueil originel des discours de Grégoire de Nazianze, traduit en arabe, qu'il a utilisé comme modèle, venait de Syrie et qu'il n'a copié que 27 Discours, au lieu des 30 habituels, créant ainsi lui-même un recueil partiel. Cette seule indication oriente déjà vers deux constatations: même si la majorité des recueils de discours de Grégoire de Nazianze actuellement disponibles permet d'identifier un recueil-type de 30 Discours, on peut en tout cas penser qu'il n'a pas été constitué tel quel dès l'origine, et qu'il a existé d'autres recueils ou d'autres groupes de Discours en Syrie, ces recueils ayant selon toute probabilité servi de modèles aux recueils arabes d'Égypte et du Sinair ${ }^{5}$, même si les recueils partiels ont parfois été complétés par d'autres discours et souvent remaniés linguistiquement.

La consultation du schéma de filiation des manuscrits ${ }^{6}$ permet déjà d'observer que la tradition manuscrite de la traduction arabe des Discours est complexe: même si deux familles de manuscrits peuvent être aisément être identifiées ( $x$ tradition sinaïtique et $y$ tradition égyptienne), certains manuscrits paraissent s'écarter partiellement de ces deux familles homogènes et soit présenter un certain nombre de contaminations, soit se rattacher à une branche parallèle dont on ne pouvait encore fixer l'origine en 1988.

Il est apparu plus tard, quand fut publiée l'édition critique de la version arabe du discours 21 (sur Athanase $)^{7}$, qu'il faut distinguer deux branches à l'intérieur de la famille $x$, l'une d'entre elles étant à proprement parler sinaïtique, l'autre étant syrienne (plusieurs manuscrits étant localisés au Liban actuel, on parlera aussi de branche syro-libanaise).

Le manuscrit $J$, qui semble souvent s'écarter de tous les autres, appartient clairement à cette branche syrienne qui se distingue de la branche sinaïtique par une acolouthie (ou ordre des discours) particulière, et des leçons qui divergent du reste de la tradition arabe de $x$ et de $y$, soit qu'elles se rapprochent des leçons de la famille grecque $n$ quand les autres manuscrits arabes $x$ et $y$ ont des leçons proches de celles de la famille grecque $m$, ou au contraire, des leçons qui se rapprochent de celles de la famille grecque $m$ quand les autres manuscrits arabes ont des leçons qui se rapprochent de celles de la famille

${ }^{5}$ Cfr. Grand'Henry, Discours 24 arabe, p. 202-203.

6 Cfr. Grand'Henry, Discours 24 arabe, p. 231.

7 Cfr. J. Grand'Henry (éd.), Sancti Gregorii Nazianzeni opera, versio arabica antiqua, I, oratio XXI (arab. 20), CCG 34, Corpus Nazianzenum 4, Turnhout 1996, p. XVII-XIX et XIII (schéma de filiation des manuscrits) (= Grand'Henry, Discours 21 arabe). 
grecque $n^{8}$. En 2000, une synthèse sur l'état de la recherche relative à la version arabe des Discours de Grégoire de Nazianze a été publiée par J. Grand'Henry et L. Tuerlinckx, assistante de recherche à l'U.C.L. Cette recherche utilise notamment les colophons (quand il y en a) des manuscrits de la version arabe des Discours. Ces colophons mentionnent que les antigraphes de plusieurs recueils de Discours sont des copies qui remontent au $\mathrm{XI}^{\mathrm{e}}$ siècle et se rattachent à la région d'Antioche, mais nous n'en avons aucune trace. Aucun des recueils arabes dits des 30 Discours ( $=29$ Discours + la Vita), que nous avons trouvés ne remonte plus haut que le XIII ${ }^{\mathrm{e}}$ siècle. Pour la période antérieure au $\mathrm{XIII}^{\mathrm{e}}$ siècle, nous n'avons trouvé que des recueils partiels (un seul pour la région du Sinaï et plusieurs d'origine syrienne). Des recueils de 15 et de 20 Discours sont signalés à Alep ${ }^{9}$ et nous avons retrouvé un recueil de 13 Discours à Alep également ${ }^{10}$ ).

$\mathrm{Si}$ on admet, ce que semble indiquer le contexte historique, que la circulation des manuscrits chrétiens s'est faite, surtout à partir de la période des croisades $\left(\mathrm{XI}^{\mathrm{e}}\right.$ siècle) et des invasions mongoles du XIII ${ }^{\mathrm{e}}$ siècle, dans le sens nord-sud, d'Antioche à Ste-Catherine du Sinaï (souci de trouver un refuge en dehors des régions perturbées par les guerres), il semble explicable que le recueil dit des 30 Discours, se soit d'une part constitué au Sinaï (famille $x$ ), et d'autre part en Égypte (famille y). Par ailleurs, il a été montré aussi que le traducteur principal, Ibrāhīm b. Yūhannā al-Antākī, qui a vécu au $\mathrm{X}^{\mathrm{e}}$ siècle, a utilisé des travaux antérieurs, et donc a été un adaptateur-réviseur autant qu'un traducteur ${ }^{11}$.

En 2001, Madame Laurence Tuerlinckx a publié l'édition critique de la version arabe des Discours $1,45,44^{12}$. Cette publication apporte un supplément d'information important sur l'histoire du texte de ces Discours de Grégoire de Nazianze et du même coup, sur la tradition arabe de l'ensemble des Discours. En effet, la présence des Discours 1, 45, 44 dans le manuscrit $W=$ Sinaï 277 ( $c a$. XII ${ }^{\mathrm{e}}$ siècle) a permis d'inclure dans l'édition critique le texte d'un manuscrit arabe plus ancien que les autres d'à peu près un siècle. Le texte de ce manuscrit se rattache clairement à la famille $x$, déjà mentionnée, et de plus, il se termine par un colophon qui précise que ce texte a été collationné (ou corrigé)

${ }^{8}$ Voir en particulier Grand'Henry, Discours 24 arabe, p. 239, note 13 ( $J$ suit le grec de $m$ ); Grand'Henry, Discours 21 arabe, p. XVIII ( $J$ suit le grec de $n$ ).

${ }^{9}$ Cfr. Grand'Henry - Tuerlinckx, Version arabe, p. 208.

${ }^{10}$ Cfr. J. Grand'Henry, Les discours de Grégoire de Nazianze et la tradition manuscrite arabe syrienne, „Le Muséon” 103 (1990) 255-265.

${ }^{11} \mathrm{Cfr}$. idem, La méthode de révision d'une version patristique arabe ancienne chez Ibrhm fils de Yhann d'Antioche, „Annales du Département de Lettres Arabes” (Université S. Joseph, Beyrouth) 6-B (1991-1992) (Hommage au Père Fiey) 161-172.

12 Cfr. L. Tuerlinckx (éd.), Sancti Gregorii Nazianzeni Opera, versio arabica antiqua, II, Orationes: I, XLV, XLIV (arab. 9, 10, 11), CCG 43, Corpus Nazianzenum 10, Turnhout 2001 (= Tuerlinckx, Discours 1, 45, 44 arabes). 
sur la base d'une copie lue selon la lecture du Protospathaire. On trouve ce terme parfois utilisé pour désigner (sans le nommer) la fonction (porte-épée) ou le titre (byzantin) porté par Ibrāhīm b. Yūhannā al-Antākī. Comme on l'a mentionné plus haut, celui-ci est connu comme traducteur/réviseur du grec en arabe des Discours ou d'une partie de ceux-ci ${ }^{13}$ (il n'est pas exclu qu'il se soit basé en partie au moins sur la version syriaque, langue que plusieurs antiochiens maîtrisaient aussi, en plus du grec et de l'arabe).

D'autre part, la présence des Discours 1, 45, 44 dans un groupe de manuscrits libanais (Balamend, Beyrouth, Dayr al-qidds Yhann Dm, St Paul à Harissa) permet de renforcer l'hypothèse de l'existence d'une famille $x$ syrosinaïtique dans laquelle le terme „syro" inclut ces manuscrits aujourd'hui libanais et proches du manuscrit $J$ d'origine syrienne dont il a été question cidessus. Comme plusieurs leçons de ces manuscrits libanais, appuyées parfois par le manuscrit $J$ et par le manuscrit $W$ cité ci-dessus sont communes, on aperçoit plus clairement que la branche syrienne de la famille syro-sinaitique remonte au moins au XII ${ }^{\mathrm{e}}$ siècle avec $W^{14}$. L'existence d'une famille intermédiaire de manuscrits arabes (entre $x$ sinaïtique et $y$ égyptienne) est nettement établie par cette édition critique ${ }^{15}$.

En 2005, nous avons publié l'édition critique de la version arabe du Discours 40 (sur le Baptême) ${ }^{16}$. Cette édition permet de faire remonter très haut (XI $\mathrm{I}^{\mathrm{e}}$ siècle) l'histoire de la branche syro-libanaise évoquée plus haut: „Pour ce qui concerne la version arabe du Discours 40, la filiation évoquée ci-dessus (= la branche syrienne ayant un lien direct avec la branche sinaïtique dans le manuscrit $W$ évoqué plus haut) trouve une confirmation remarquable, même si la situation est moins claire quant aux rapports entre $J$ et $N$, ainsi qu'on l'a indiqué plus haut, et si le 'maillon manquant' $W$ n'existe pas pour le Discours 40. En effet, l'ensemble de la tradition manuscrite s'y divise entre un groupe $M i$ (XI ${ }^{\mathrm{e}} \mathrm{s}$.) $J Y$ qu'il paraît justifié d'appeler 'proto-version syrienne' et un groupe FONG EHI PU DQ dans lequel on retrouve les familles sinaitique $x$, égyptienne $y$, et intermédiaire $z^{\prime 17}$.

Un fait d'importance majeure pour l'histoire du texte caractérise notamment cette proto-version syrienne: alors que certains manuscrits arabes de la famille sinaïtique $x(F O N G)$, de la famille intermédiaire $z(P U)$ et de la famille égyptienne $y$ ( $E H I D Q)$ ont une omission significative dans le texte arabe correspondant au grec de la $P G 364$ B 11-13 = SC 358, p.04 1. 10-12: ,la

${ }^{13}$ Cfr. Tuerlinckx, Discours 1, 45, 44 arabes, p. XI-XII.

${ }^{14}$ Cfr. Tuerlinckx, Discours 1, 45, 44 arabes, p. XXIV.

${ }^{15}$ Cfr. Tuerlinckx, Discours 1, 45, 44 arabes, p. XXIX-XXXII.

${ }^{16}$ Cfr. J. Grand'Henry (éd.), Sancti Gregorii Nazianzeni Opera, versio arabica antiqua, III, Oratio XL (arab. 4), CCG 57, Corpus Nazianzenum 19, Turnhout 2005 (= Grand'Henry, Discours 40 arabe, p. XII).

17 Cfr. Grand'Henry, Discours 40 arabe, p. XII. 
deuxième lumière, c'est l'ange qui est un sorte d'écoulement de la première lumiè re ou de participation à elle; parce qu'il a une propension vers elle, et qu'elle le soutient, il tient d'elle son illumination (traduction de P. Gallay dans les Sources Chrétiennes)".Le passage en caractères italiques est le passage omis dans les manuscrits arabes signalés ci-dessus. Seule la proto-version syrienne donne un équivalent arabe complet de ce passage.

On signalera aussi une particularité notable de la version arabe du Discours 40 sur le plan doctrinal: on sait qu'au chapitre 43 du discours grec, Grégoire de Nazianze affirme que „le Père est plus grand $(\mu \varepsilon i \zeta \omega v)$, ce qui „n'a pas d'autre sens que d'être à l'origine des Personnes, qui sont cependant égales au Père"18. Cependant, „l'affirmation que le Père est plus grand que le Fils (souligné par nous), puisque le Fils s'est incarné", se trouve chez Ambroise ${ }^{19}$. Or, cette affirmation selon laquelle le Père est plus grand que le Fils, se trouve dans tous les manuscrits de la version arabe du Discours $40^{20}$. Le Discours 40 en grec n'ayant pas encore été collationné par notre équipe de Louvain-la-Neuve, il n'est pas encore possible de préciser dans l'état actuel de la recherche quels sont les manuscrits grecs qui auraient la même formule que celle d'Ambroise de Milan et des manuscrits de la version arabe.

Dans les premières publications du ,groupe de recherches sur les Discours de Grégoire de Nazianze" de Louvain-la-Neuve, une incertitude planait encore sur la signification réelle à accorder à la plus grande proximité de la branche syrienne des manuscrits arabes avec le texte grec originel. Du fait que ce groupe n'avait à sa disposition à cette époque que le seul manuscrit $J$ comme représentant de cette branche dans les Discours édités alors, et qu'il s'agissait d'un manuscrit tardif (XVIII ${ }^{\mathrm{e}}$ siècle), la question de savoir si le texte de $J$ n'avait pas été l'objet d'un remaniement tardif sur la base d'une édition imprimée du grec, se posait encore. Aujourd'hui, il apparaît que la branche syrienne fournit, d'une façon générale et grâce au fait que nous possédons maintenant des copies de beaucoup de manuscrits syro-libanais, un texte relativement proche du grec originel, plus proche en tout cas que celui des manuscrits de la famille $x$ sinaitique, $y$ égyptienne et $z$ intermédiaire. Seule la publication future des autres Discours de la version arabe non encore édités permettra d'affiner ces analyses.

Il apparaît en tout cas d'ores et déjà que la question du rapport entre les grandes familles de manuscrits grecs de Grégoire telles qu'elles ont été identifiées par les savants polonais de l'Académie des Sciences de Cracovie (Th. Sinko et J. Sajdak surtout, mais aussi L. Sternbach, St. Witkowski, G. Przy-

${ }^{18}$ Cfr. C. Moreschini (éd.) et P. Gallay (traducteur), Grégoire de Nazianze, Discours 38-41, Introduction, texte critique et notes, SCh 358, Paris 1990, 43.

${ }^{19}$ Cfr. Ambrosius, De fide II 8, 59, IV 12, 168, CSEL 78, 77 et 216.

20 Cfr. Grand'Henry, Discours 40 arabe, p. VIII, XVI-XVIII et p. 175, 1. 1. 
chocki et G. Hammer), à savoir les familles $m$ et $n$, complétées par le $x$ des manuscrits grecs n'appartenant ni à $m$, ni à $n^{21}$ et la version arabe, est très complexe. Sur ce sujet, et sur bien d'autres encore, la thèse doctorale en préparation de notre collaboratrice fidèle, Madame L. Tuerlinckx, apportera de précieux éclaircissements ${ }^{22}$.

C'est avec un plaisir particulier que nous offrons cette contribution en hommage déférent et cordial au Professeur Marek Starowieyski, rencontré pour la première fois au Deuxième Symposium Nazianzenum réuni à Louvain-la-Neuve en 1981, et avec qui nous n'avons pas cessé d'entretenir des rapports de collégialité et d'amitié depuis 25 ans.

${ }^{21}$ Cfr. V. Somers, Histoire des collections complètes des „Discours” de Grégoire de Nazianze (Publications de l'Institut Orientaliste de Louvain 48), Louvain-la-Neuve 1997.

22 Cfr. L. Tuerlinckx, Histoire de la version arabe de Grégoire de Nazianze. Édition critique des Discours 27 et 38 (avec analyse linguistique et index lemmatisé). Étude de la tradition manuscrite et identification des divers états du texte (traduction et révision), en préparation (octobre 2006). Pour un inventaire exhaustif avec mise à jour régulière des travaux du Centre Grégoire de Nazianze de Louvain-la-Neuve, voir le site web: http://nazianzos.fltr.ucl.be 